\title{
DECOMPOSITION FORMULAS FOR A CLASS OF PARTIAL DIFFERENTIAL EQUATIONS
}

\author{
ABDULLAH ALTIN AND EUTIQUIO C. YOUNG
}

\begin{abstract}
The paper presents decomposition formulas for solutions of a class of singular partial differential equations. The equations consist of products of iterated differential operators each of which involves a real parameter. The decomposition is given in terms of solutions corresponding to each operator.
\end{abstract}

1. Introduction. In this paper we present decomposition formulas of solutions of a class of partial differential equations of the form

$$
\left(\prod_{i=1}^{p} L_{\alpha_{i}}^{m_{i}}\right) u \equiv\left(L_{\alpha_{1}}^{m_{1}} L_{\alpha_{2}}^{m_{2}} \cdots L_{\alpha_{p}}^{m_{p}}\right) u=0
$$

where

$$
L_{\alpha_{i}}=\frac{\partial^{2}}{\partial y^{2}}+\frac{\alpha_{i}}{y} \frac{\partial}{\partial y}+P .
$$

$P$ being a general linear operator of arbitrary order $r$ which satisfies

$$
\frac{\partial}{\partial y} P(u)=P\left(\frac{\partial u}{\partial y}\right)
$$

The constants $\alpha_{i}$ are real parameters and $m_{i}$ are positive integers, $1 \leqslant i \leqslant p$. The domain of each of the operators $L_{\alpha_{i}}(1 \leqslant i \leqslant p)$ is the set of all real valued functions $u(x, y)$ of class $C^{2}(0, \infty) \cap C^{r}(D)$, where $x=\left(x_{1}, x_{2}, \ldots, x_{n}\right)$ and $D$ is the regularity domain of $u$ in $R^{n}$. We denote a solution of $L_{\alpha} u=0$ by $u^{\alpha}(x, y)$ or simply by $u\{\alpha\}$. As was pointed out by Weinstein [6], solutions of the equation $L_{\alpha} u=0$ satisfy the recursive relations

$$
\begin{aligned}
& \text { (a) } u^{\alpha}(x, y)=y^{1-\alpha} u^{2-\alpha}(x, y), \\
& \text { (b) } u_{y}^{\alpha}(x, y)=y u^{\alpha+2}(x, y)
\end{aligned}
$$

When $P= \pm \Delta$, the $n$-dimensional Laplacian, $\alpha_{i}=\alpha$ and $m_{i}=1,1 \leqslant i \leqslant p$, several decomposition formulas for solutions of (1) are well known. See, for example, Weinstein [7] and Payne [2]. For the iterated wave equation, corresponding results were obtained by Krahn [1]. Such representation formulas have proved useful in certain physical problems, such as the study of axially symmetric Stokes' flow of a viscous fluid (cf. Payne and Pell [3, 4, 5]).

Received by the editors May 19, 1981

1980 Mathematics Subject Classification. Primary 35A25, 35C99.

Key words and phrases. Decomposition formula, recursive relation, iterated Laplacian, general solution. 
In [8], [9] Weinstein established his formula by solving a certain boundary value problem for the Laplace operator $\Delta$. Our method here relies only on the recursive relation (3b) and, thus, appears much simpler.

2. A decomposition formula. We first establish the following lemma.

Lemma 1. Let $u\{\beta\} \in C^{2 m(p)}(0, \infty) \cap C^{r m(p)}(D)$ satisfy the equation $L_{\beta} u=0$. Then

$$
\left(\prod_{i=1}^{p} L_{\alpha_{i}}^{m_{i}}\right) u\{\beta\}
$$

$$
=\prod_{i=1}^{p} \prod_{j=0}^{m_{i}-1}\left(\alpha_{i}-2 j-2[m(p)-m(i)]-\beta\right) u\{\beta+2 m(p)\}
$$

where $m(i)=m_{1}+m_{2}+\cdots+m_{i}, 1 \leqslant i \leqslant p$.

Proof. From the definition we have

$$
\begin{aligned}
L_{\alpha} u\{\beta\} & =\left(\frac{\partial^{2}}{\partial y^{2}}+\frac{\beta+(\alpha-\beta)}{y} \frac{\partial}{\partial y}+P\right) u\{\beta\} \\
& =L_{\beta} u\{\beta\}+\frac{\alpha-\beta}{y} \frac{\partial}{\partial y} u\{\beta\} .
\end{aligned}
$$

By (3b) this can be written as

$$
L_{\alpha} u\{\beta\}=(\alpha-\beta) u\{\beta+2\} .
$$

Applying the operator $L_{\alpha}$ repeatedly on both sides of (5), we then obtain by induction the result

$$
L_{\alpha}^{m} u\{\beta\}=\prod_{j=0}^{m-1}(\alpha-2 j-\beta) u\{\beta+2 m\} .
$$

Now replace $\alpha$ and $m$ in (6) by $\alpha_{p}$ and $m_{p}$, respectively, and apply in succession the operators $L_{\alpha_{p-1}}^{m_{p-1}}, L_{\alpha_{p-2}}^{m_{p-2}}, \ldots, L_{\alpha_{1}}^{m_{1}}$ on both sides of (6). By induction we readily obtain the formula (4).

Using Lemma 1 we can now prove our first theorem.

THEOREM 1. Let the parameters $\alpha_{i}(1 \leqslant i \leqslant p)$ satisfy the condition

$$
\begin{aligned}
\alpha_{r}-\alpha_{s} \neq 2[m(s-1)- & m(r)+1]+2 \nu \\
& \left(\nu=0,1, \ldots, m_{r}+m_{s}-2,1 \leqslant r<s=2,3, \ldots, p\right) .
\end{aligned}
$$

Then the general solution of equation (1) is given by the formula

$$
u(x, y)=\sum_{i=1}^{p} \sum_{j=0}^{m_{i}-1} u\left\{\alpha_{i}-2 j-2[m(p)-m(i)]\right\} .
$$

We observe that the only arbitrariness in the decomposition (8) is that each term on the right-hand side of (8) may be determined up to a function $v(x)$ which satisfies $P v=0$ in $D$ such that their sum $\Sigma v$ is identically zero. 
To prove the theorem, set

$$
\beta_{i j}=\alpha_{i}-2 j-2[m(p)-m(i)]
$$

for $1 \leqslant i \leqslant p$ and $0 \leqslant j \leqslant m_{i}-1$. Under the condition (7), we see that the $\beta_{i j}$ 's are all distinct. In fact, consider

$$
\begin{aligned}
\beta_{r j}-\beta_{s k} & =\alpha_{r}-\alpha_{s}-2(j-k)-2[m(s)-m(r)] \\
& =\alpha_{r}-\alpha_{s}-2[m(s-1)-m(r)+1]-2\left[\left(m_{s}-1\right)+j-k\right] \\
& =\alpha_{r}-\alpha_{s}-2[m(s-1)-m(r)+1]-2 \nu
\end{aligned}
$$

where $\nu=m_{s}-1+j-k$. Since $0 \leqslant j \leqslant m_{r}-1$ and $0 \leqslant k \leqslant m_{s}-1, \nu$ ranges from 0 to $m_{s}+m_{r}-2$. Thus it follows from (7) that $\beta_{r j} \neq \beta_{s k}$ for $r \neq s$. This implies that as $i$ ranges from 1 to $p$ and $j$ from 0 to $m_{i}-1$, we have $m_{1}+\cdots+m_{p}$ $=m(p)$ distinct values of (9). Therefore in (8) we have the correct number of functions $u\left\{\beta_{i j}\right\}$.

By setting $\beta=\beta_{i j}$, it follows from Lemma 1 that

$$
\left(\prod_{i=1}^{p} L_{\alpha_{i}}^{m_{i}}\right) u\left\{\beta_{i j}\right\}=0 \text {. }
$$

Since the differential operator is linear, we conclude that $\sum_{i=1}^{p} \sum_{j=0}^{m_{i}-1} u\left\{\beta_{i j}\right\}$ also satisfies (1) and thus provides the general solution of the equation.

When $P$ is the $n$-dimensional Laplacian and $m_{i}=1,1 \leqslant i \leqslant p$, the formula (8) reduces to the representation

$$
u=u^{\alpha_{p}}+u^{\alpha_{p-1}-2}+\cdots+u^{\alpha_{1}-2(p-1)} \quad\left(\alpha_{r}-\alpha_{s} \neq 2(s-r), r<s=2, \ldots, p\right)
$$

obtained by Weinstein (7).

3. Further result. Here we present a decomposition formula for solutions of a special case of (1) in which the condition (7) on the parameters are partly violated. We first establish the following lemma.

LEMMA 2. Let $p$ and $m_{1}, m_{2}, \ldots, m_{p}$ be positive integers. Then

$$
\begin{aligned}
&\left(\prod_{i=1}^{p} \prod_{j=1}^{m_{i}} L_{\alpha_{i}+2\left(m_{i}-j\right)}\right) u\{\beta\} \\
&=\prod_{i=1}^{p}\left(\alpha_{i}-2[m(p)-m(i)]-\beta\right)^{m_{i}} u\{\beta+2 m(p)\} .
\end{aligned}
$$

Proof. From (5) we have $L_{\alpha} u\{\beta\}=(\alpha-\beta) u\{\beta+2\}$. If we again apply $L_{\alpha+2}$ on both sides of the equation we find

$$
\begin{aligned}
L_{\alpha+2} L_{\alpha} u\{\beta\} & =(\alpha-\beta) L_{\alpha+2} u\{\beta+2\} \\
& =(\alpha-\beta)[(\alpha+2)-(\beta+2)] u\{(\beta+2)+2\} \\
& =(\alpha-\beta)^{2} u\{\beta+4\} .
\end{aligned}
$$

Hence by induction we find

$$
\left(\prod_{j=1}^{m} L_{\alpha+2(m-j)}\right) u\{\beta\}=(\alpha-\beta)^{m} u\{\beta+2 m\} .
$$


Now replace $\alpha$ and $m$ by $\alpha_{p}$ and $m_{p}$, respectively, and apply the operator $\Pi_{j=1}^{m} \underline{p}_{1}^{-1} L_{\alpha_{p-1}}+2\left(m_{p-1}-j\right)$ to both sides of (11). We obtain

$$
\begin{aligned}
\left(\prod_{i=p-1}^{p} \prod_{j=1}^{m_{i}} L_{\alpha_{i}+2\left(m_{i}-j\right)}\right) u\{\beta\} \\
\quad=\left(\alpha_{p}-\beta\right)^{m_{p}}\left(\prod_{j=1}^{m_{p-1}} L_{\alpha_{p-1}+2\left(m_{p-1}-j\right)}\right) u\left\{\beta+2 m_{p}\right\} \\
=\left(\alpha_{p}-\beta\right)^{m_{p}}\left(\alpha_{p-1}-\left[\beta+2 m_{p}\right]\right)^{m_{p-1}} u\left\{\left(\beta+2 m_{p}\right)+2 m_{p-1}\right\} \\
=\prod_{i=p-1}^{p}\left(\alpha_{i}-2[m(p)-m(i)]-\beta\right)^{m_{i}} u\left\{\beta+2\left(m_{p}+m_{p-1}\right)\right\} .
\end{aligned}
$$

By similarly applying the operators $\prod_{j=1}^{m_{i}} L_{\alpha_{i+2\left(m_{i}-j\right)}}$, for $i=p-2, \ldots, 3,2,1$ on (12) we then obtain the formula (10).

THEOREM 2. Let the parameters $\alpha_{i}, 1 \leqslant i \leqslant p$, satisfy the condition

$$
\alpha_{r}-\alpha_{s} \neq 2[m(s)-m(r)], \quad r<s=2, \ldots, p .
$$

Then the general solution of the equation

$$
\left(\prod_{i=1}^{p} \prod_{j=1}^{m_{i}} L_{\alpha_{i}+2\left(m_{i}-j\right)}\right) u=0
$$

is given by the formula

$$
u(x, y)=\left.\sum_{i=1}^{p} \sum_{j=0}^{m_{i}-1} \frac{\partial^{j} u\{\beta\}}{\partial \beta^{j}}\right|_{\beta=\beta_{i}}
$$

where $\beta_{i}=\alpha_{i}-2[m(p)-m(i)]$ and $\partial^{0} u\{\beta\} / \partial \beta^{0}=u\{\beta\}$.

Here we note that for each $m_{i}, 1 \leqslant i \leqslant p$, the parameters $\alpha_{i}+2\left(m_{i}-j\right), 1 \leqslant j \leqslant$ $m_{i}$, violate the condition (7). This accounts for the appearance of derivatives in (15).

The proof is similar to that of the processing theorem. Under the condition (13), the quantities $\beta_{i}, 1 \leqslant i \leqslant p$, are all distinct. From Lemma 2 we have

$$
\prod_{i=1}^{p}\left(\prod_{j=1}^{m_{i}} L_{\alpha_{i}+2\left(m_{i}-j\right)}\right) u\{\beta\}=\prod_{i=1}^{p}\left(\beta_{i}-\beta\right)^{m_{i}} u\{\beta+2 m(p)\} .
$$

Hence, for $j=0,1, \ldots, m_{i}-1$, each of the functions $\left.(\partial / \partial \beta)^{j} u\{\beta\}\right|_{\beta=\beta_{i}}$ satisfies the equation

$$
\prod_{i=1}^{p}\left(\prod_{j=1}^{m_{i}} L_{\alpha_{i}+2\left(m_{i}-j\right)}\right) u=0
$$

and by linearity so does their sum. This establishes the formula (15).

We remark that the method employed above can be applied as well to consider equation (1) in the case when the condition (7) is violated for some $r \neq s$. When $P=\Delta$ and $m_{i}=1,1 \leqslant i \leqslant p$, a representation formula for such a case was obtained by Payne [2] through a limiting process. We illustrate this case by means of an example given below. 
EXAMPLE 1. For $\alpha, \lambda, \delta$ any real constants, consider the equation $L_{\delta}^{3} L_{\lambda}^{4} L_{\alpha}^{2}(u)=0$. Then by Lemma 1,

$$
\begin{aligned}
L_{\delta}^{3} L_{\lambda}^{4} L_{\alpha}^{2} u\{\beta\}= & (\alpha-\beta)(\alpha-2-\beta)(\lambda-4-\beta) \\
& \times(\lambda-6-\beta)(\lambda-8-\beta)(\lambda-10-\beta) \\
& \times(\delta-12-\beta)(\delta-14-\beta)(\delta-16-\beta) u\{\beta+18\} .
\end{aligned}
$$

Hence if we choose $\alpha=3, \lambda=-1, \delta=14$ which satisfy the condition (7), then by Theorem 1, the general solution of the equation $L_{14}^{3} L_{-1}^{4} L_{3}^{2}(u)=0$ is given by the formula

$$
\begin{aligned}
u(x, y)= & u\{3\}+u\{1\}+u\{-5\}+u\{-7\}+u\{-9\} \\
& +\{-11\}+u\{2\}+u\{0\}+u\{-2\} .
\end{aligned}
$$

On the other hand, if $\alpha=3, \lambda=5, \delta=15$, so that the condition (7) is violated, then equation (16) gives

$$
L_{15}^{3} L_{5}^{4} L_{3}^{2} u\{\beta\}=(3-\beta)^{2}(1-\beta)^{3}(-1-\beta)^{2}(-3-\beta)(-5-\beta) u\{\beta+18\} .
$$

Thus we see that $\left.(\partial / \partial \beta)^{k} u\{\beta\}\right|_{\beta=\beta_{j}}$ satisfy the equation. Hence the general solution of $L_{15}^{3} L_{5}^{4} L_{3}^{2}(u)=0$ is given by

$$
\begin{aligned}
u(x, y)= & u\{3\}+u^{\prime}\{3\}+u\{1\}+u^{\prime}\{1\}+u^{\prime \prime}\{1\} \\
& +u\{-1\}+u^{\prime}\{-1\}+u\{-3\}+u\{-5\}
\end{aligned}
$$

where

$$
u^{(k)}\left\{\beta_{j}\right\}=\left.\left(\frac{\partial}{\partial \beta}\right)^{(k)} u\{\beta\}\right|_{\beta=\beta_{j}}
$$

EXAMPLE 2. For $\alpha, \lambda, \delta$ any real constants, consider the equation

$$
L_{\delta+4} L_{\delta+2} L_{\delta} L_{\lambda+2} L_{\lambda} L_{\alpha+6} L_{\alpha+4} L_{\alpha+2} L_{\alpha}(u)=0 .
$$

Then by Lemma 2,

$$
\begin{aligned}
& L_{\delta+4} L_{\delta+2} L_{\delta} L_{\lambda+2} L_{\lambda} L_{\alpha+6} L_{\alpha+4} L_{\alpha+2} L_{\alpha} u\{\beta\} \\
&=(\alpha-\beta)^{4}(\lambda-8-\beta)^{2}(\delta-12-\beta)^{3} u\{\beta+18\} .
\end{aligned}
$$

Hence for $\alpha \neq \lambda-8 \neq \delta-12$, the general solution of the equation is

$$
\begin{aligned}
u(x, y)= & u\{\alpha\}+u^{\prime}\{\alpha\}+u^{\prime \prime}\{\alpha\}+u^{\prime \prime \prime}\{\alpha\}+u\{\lambda-8\} \\
& +u^{\prime}\{\lambda-8\}+u\{\delta-12\}+u^{\prime}\{\delta-12\}+u^{\prime \prime}\{\delta-12\} .
\end{aligned}
$$

If $\alpha=\lambda-8=\delta-12$, then it is obvious that the general solution of the given equation is

$$
u(x, y)=u\{\alpha\}+u^{\prime}\{\alpha\}+\cdots+u^{(8)}\{\alpha\}
$$

\section{REFERENCES}

1. D. Krahn, On the iterated wave equation, Nederl. Akad. Wetensch. Proc. Ser. A 60 (1957), 492-505.

2. L. E. Payne, Representation formulas for solutions of a class of partial differential equations, J. Math. Phys. 38 (1959), 145-149.

3. L. E. Payne and W. H. Pell, The Stokes flow problem for a class of axially symmetric bodies, J. Fluid Mech. 7 (1960), 529-549. 
4. __ The Stokes flow about a spindle, Quart. Appl. Math. 18 (1960), 257-262.

5. On Stokes flow about a torus, Mathematica 7 (1960), 78-92.

6. A. Weinstein, The generalized radiation problem and the Euler-Poisson-Darboux equation, Summa Brasil. Math. 3 (1955), 125-147.

7. $245-254$.

8. __ Generalized axially symmetric potential theory, Bull. Amer. Math. Soc. 59 (1953), 20-38.

9. On the wave equation and the equation of Euler-Poisson, Proc. Sympos. Appl. Math., Vol. 5, Amer. Math. Soc., Providence, R.I., 1954, pp. 137-147.

Faculty of Science, University of ANKara, BeSEVler, ANKara, Turkey

Department of Mathematics, Florida State University, Tallahassee, Florida 32306 